\title{
Editorial
}

\section{SIDE-EFFECTS OF ANTILEPROSY DRUGS IN COMMON USE}

\section{Introduction}

The drugs which I shall describe are dapsone, clofazimine, rifampicin, ethionamide and thiacetazone. I have chosen ethionamide rather than prothionamide, as the latter is no longer marketed in Britain although still available in some countries. This choice matters little, for these two closely related drugs are equally effective in leprosy, and the reported reduced incidence of gastric side-effects from prothionamide (which my own experience does not confirm) is offset by the lower cost of ethionamide. Before describing side-effects I must draw attention to two points. First, the difficulty in deciding which drug is responsible when multidrug therapy is being administered, especially as certain combinations of drugs are known to act synergistically, and this fact should be given consideration when side-effects of rifampicin, ethionamide and thiacetazone are discussed. Secondly, although reactions (reactional states) can be precipitated by chemotherapy, I propose to exclude them in this paper as the relationship is indirect and, furthermore, considerations of space are involved.

I describe side-effects under two headings in order to separate those which are mild from those of greater significance. Where I use the term 'gastro-intestinal symptoms' I include any or all of the following: anorexia, nausea, vomiting, abdominal discomfort and disturbed bowel function, and the term 'transient rash' implies an irritating erythematous rash, of ten localized to face and neck, but which may be generalized. All these symptoms are usually self-limiting. As regards serious side-effects, I shall describe in these introductory pages the clinical and laboratory features of those which are produced by more than one of these 5 drugs, and thus avoid repetition; details of those which occur with only one of them will be described in the appropriate sections. It should be noted that recovery is the general rule if the offending drug is promptly withdrawn.

\section{A EFFECTS ON SKIN}

The following skin complications may occur as part of a hypersensitivity 
syndrome which includes fever, eosinophilia, lymphadenopathy and hepatitis, or may occur on their own as an incomplete form of the syndrome.

\section{Exanthematous skin eruption}

This is a generalized skin eruption, generally symmetrical but often the face is spared. It may simulate scarlatina or measles, or may consist of small erythematous papules or urticarial weals. Exfoliative dermatitis may supervene.

\section{Exfoliative dermatitis}

The patient feels cold and shivery, and temperature is raised. The entire skin, including that of the scalp, flakes off as scales and leaves a pronounced redness.

\section{Toxic epidermal necrolysis (Lyell's syndrome; the scalded skin syndrome)}

This is ushered in by malaise and fever. The skin feels tender and erythema begins in axillae and groins, extending over the entire skin surface excepting the scalp. The erythema may become papular and may irritate. Large flaccid blisters, filled with clear fluid, appear on face, trunk and limbs, and large areas of skin become detached as if scalded. A few days later the detached skin peels off in large sheets, revealing pink, oozing, painful areas. Death has been recorded as a rare event if the drug has not been withdrawn.

\section{Stevens-Johnson syndrome (erythema multiforme bullosum)}

This is a severe bullous form of erythema multiforme in which bullae of skin and oral mucosa are associated with fever and prostration. Bullae of mouth ulcerate and become crusted, while eyes may be involved in the form of conjunctivitis and corneal ulceration. The illness is likely to last for 2 or 3 weeks and carries a mortality of about $5-15 \%$.

EFFECT ON BLOOD

\section{Haemolytic anaemia}

A severe haemolysis will be suspected by pallor and slight jaundice. Laboratory findings include a marked reduction in haemoglobin and in haptoglobulin concentration, red blood cells are normochromic and show anisocytosis, poikilocytosis, and may contain Heinz bodies. Their lifespan is reduced. There is an increase in reticulocytes, and also in serum bilirubin and urinary urobilinogen. 


\section{Agranulocytosis}

This should be suspected in a patient who develops fever, fatigue, headache and sore throat. The pharynx becomes ulcerated and there is a severe shortage of granulocytes in the blood.

\section{Thrombocytopenia}

There is an increased bleeding tendency in the form of petechiae or purpura, or less commonly of haemorrhagic bullae of oral mucosa or of bleeding from bowel or urinary tract. Thrombocytes (platelets) are much reduced.

\section{EFFECTS ON NER VES}

\section{Peripheral neuropathy}

Classical findings are weakness in muscles, impaired sensation in limbs, and weak or absent tendon reflexes. These signs are bilateral and may be confined to upper or to lower limbs, or may affect both. Early manifestations are clumsiness in walking or in using the hands, and bilateral footdrop is a common sequel.

\section{EFFECTS ON BRAIN}

\section{Psychosis}

Symptoms include insomnia, irritability, agitation, violence, delusions, disorientation, visual and auditory hallucinations, and speech which is slow and incoherent.

\section{E EFFECTS ON LIVER}

\section{Hepatitis}

This may occur as part of a hypersensitivity syndrome of fever, eosinophilia, lymphadenopathy, and any one of the hypersensitivity skin manifestations which have already been described, or it may occur on its own as an incomplete form of the syndrome. Hepatitis is heralded by malaise, anorexia, nausea, discomfort in muscles and joints, darkening of urine, and a faint yellowness of conjunctivae. Later developments are jaundice, dark urine, tender liver enlargement, bilirubinaemia, and abnormal liver function tests. 
F EFFECTS ON KIDNEYS

\section{Renal failure}

Prodromal symptoms may occur soon after ingestion of the drug, disappearing within a few hours, and may be repeated on several occasions before oliguria is noticed. These are malaise, chilliness, nausea, vomiting, mild pyrexia, and lumbar discomfort. Protein is found in urine, plasma urea and creatinine are raised, and oliguria after a few days is replaced by anuria.

\section{Five antileprosy drugs and their side-effects (Table 1)}

\section{DAPSONE (DDS; $4: 4^{\prime}$ DIAMINODIPHENYL SULPHONE)}

(a) Mild side-effects

These include gastro-intestinal symptoms, transient rash, headache, dizziness, and a sensation of 'woolly-headedness' - an inability to think clearly for a few hours after taking the tablet. Methaemoglobinaemia (causing a faint cyanosis) and a mild haemolysis commonly occur and are harmless.

\section{(b) Serious side-effects}

Because dapsone is effective in a number of dermatological disorders and often has to be used in dosage greater than $100 \mathrm{mg} /$ day, serious side-effects are almost exclusively encountered by dermatologists. These are as follows:

(1) Haemolytic anaemia. This may occur when dosage is $200 \mathrm{mg}$ /day or more; smaller dosage may be responsible in persons who are deficient in the enzyme glucose-6-phosphate dehydrogenase (G-6-PD). But deficiency of this enzyme is not the only factor, for a toxic dapsone derivative (DDS-NHOH) has been implicated. It is noteworthy that a mild haemolytic anaemia has been induced in an infant by dapsone transmitted in breast milk; the infant had significant serum concentrations of dapsone.

(2) Agranulocytosis. This is a manifestation of marrow depression, and although a few cases have been reported by dermatologists using dapsone monotherapy, in most of the reported cases dapsone has been used in combination with other drugs in the prophylaxis of malaria.

(3) Hypersensitivity. This is confined to the first 6 weeks of treatment, and manifestations include some or all of the following: fever, eosinophilia, mononucleosis, lymphadenopathy, hepatitis, and an exanthematous skin eruption which may progress to one of the dermatological emergencies which I have already described (exfoliative dermatitis, toxic epidermal necrolysis, and the Stevens-Johnson syndrome). A fatal hypersensitivity reaction known as 'DDS 
Table 1. Significant side-effects of five antileprosy drugs

\begin{tabular}{|c|c|c|c|c|c|c|}
\hline & Side-effect & DDS & B663 & RFP & ETH & TBI \\
\hline Adrenals & Adrenal crisis & & & Yes & & \\
\hline \multirow{3}{*}{ Blood } & Agranulocytosis & Yes & & & & Yes \\
\hline & Haemolytic anaemia & Yes & & Yes & & Yes \\
\hline & Thrombocytopenia & & & Yes & & Yes \\
\hline Bones & Osteomalacia & & & Yes & & \\
\hline \multirow[t]{2}{*}{ Bowel } & Eosinophilic enteritis & & Yes & & & \\
\hline & 'Small bowel syndrome' & & Yes & & & \\
\hline \multirow[t]{2}{*}{ Brain } & Psychosis & Yes & & Yes & & \\
\hline & Pellagra-like encephalopathy & & & & Yes & \\
\hline \multirow[t]{3}{*}{ Kidneys } & Nephrotic syndrome & Yes & & & & \\
\hline & Renal failure & & & Yes & & \\
\hline & Renal papillary necrosis & Yes & & & & \\
\hline Liver & Hepatitis & Yes & & Yes & Yes & Yes \\
\hline Nerves & Peripheral neuropathy & Yes & & & Yes & \\
\hline \multirow[t]{8}{*}{ Skin } & Exanthematous eruption & Yes & & & & Yes \\
\hline & Exfoliative dermatitis & Yes & & & & Yes \\
\hline & Fixed drug eruption & Yes & & & & \\
\hline & Ichthyosis & & Yes & & & \\
\hline & Pemphigus vulgaris & & & Yes & & \\
\hline & Porphyria cutanea tarda & & & Yes & & \\
\hline & Stevens-Johnson syndrome & Yes & & Yes & & Yes \\
\hline & Toxic epidermal necrolysis & Yes & & & & Yes \\
\hline Thyroid & Hypothyroidism & & & & Yes & \\
\hline \multirow[t]{6}{*}{ Miscellaneous } & 'DDS syndrome' & Yes & & & & \\
\hline & 'Flu' syndrome & & & Yes & & \\
\hline & Gynaecomastia & & & & Yes & \\
\hline & Haemorrhagic disease of newborn & & & Yes & & \\
\hline & Hypoalbuminaemia & Yes & & & & \\
\hline & Hypoglycaemia & & & & Yes & \\
\hline
\end{tabular}

syndrome' was described by leprologists in the early years of the drug's use. It disappeared during the decades when low dosage was in vogue, only to reappear as a rare phenomenon in recent years. One such case has been reported in a patient taking $100 \mathrm{mg} /$ day. After 3 weeks he complained of malaise, myalgia, and a rash on abdomen. A few days later he developed fever, slight jaundice, sore throat, and an erythematous maculopapular rash covering the entire body. Clinical examination revealed exudative pharyngotonsilitis, generalized tender lymphadenopathy, hepatosplenomegaly, and epigastric tenderness. The number of white blood cells steadily increased to $72,800 \mathrm{~mm}^{3}$ with $28 \%$ eosinophils. Skin biopsy was consistent with lepromatous leprosy and erythema multiforme. Death was ascribed to an extreme hypersensitivity reaction to dapsone. 
(4) Fixed drug eruption. This takes the form of one or more sharply marginated round or oval erythematous macules which become violet, brown or black, and persist for a long time.

(5) Peripheral neuropathy. This has been reported by dermatologists, and clearly could pose a diagnostic problem in leprosy. However, the diagnosis could be suspected if there were no tender nerves on palpation and if tendon reflexes were depressed or absent.

(6) Psychosis. To attribute this complication to dapsone could be difficult when treatment is administered in a leprosarium, for the incidence of psychosis in segregated patients is about $10 \%$. However, the role of dapsone in causing psychosis has been established as a rare occurrence in out-patients.

(7) Hypoalbuminaemia. This is a very rare side-effect which has never been encountered in leprosy. The few reports have been by dermatologists in patients who have received dapsone over many years for dermatitis herpetiformis. Symptoms include dyspnoea, pleural effusion, peripheral oedema, polyuria, and ascites, and tests show hypoalbuminaemia. The syndrome is attributed to great increase in intravascular albumin catabolism and a modest decrease in synthesis. There has been one death in the 5 cases described to date.

(8) Nephrotic syndrome. The case has been reported of a patient suffering from a 'pruritic skin eruption' receiving dapsone $100 \mathrm{mg} /$ day for 3 weeks and developing massive proteinuria, hypoproteinaemia, oedema and lipidaemia. Recovery was uneventful.

(9) Renal papillary necrosis. A patient suffering from dermatitis herpetiformis who had received fairly large daily doses of dapsone for 15 years began having attacks of renal colic, and 5 years later was found to have bilateral renal papillary necrosis. A blood count showed a moderate haemolytic anaemia and reticulocytosis.

\section{CLOFAZIMINE (B 663; LAMPRENE)}

A bright red iminophenazine dye.

\section{(a) Mild side-effects}

These are dose-related and reversible, the commonest being a red-brown colouration of skin and mauve or brownish-black pigmentation of skin lesions. These changes are scarcely noticeable in dark skins, but light-skinned persons find them unacceptable, and some of my patients have complained that dark marks on face and limbs have embarrassed them for 5 years after stopping the drug. Conjunctivae show varying degrees of red-brown pigmentation, and redness may be seen in urine, faeces, sputum, sweat and tears during treatment. Less common side-effects a re gastro-intestinal symptoms and a general dryness of the skin which may progress to ichthyosis of limbs. Less common still are other 
dermal manifestations such as transient rash, acne, itching or burning discomfort in skin lesions, and phototoxicity (increased reactivity of skin to light). There is one report of a reversible ocular side-effect in the form of brownish pigmentation in cornea and macula.

\section{(b) Serious side-effects}

These are confined to the small bowel and are dose-related. An early syndrome commences within a few days or weeks of instituting treatment and consists of gastro-intestinal symptoms which can be relieved by reducing dosage. A late syndrome is of more serious significance. It begins after months or years of high dosage of clofazimine, and a good name for it would be 'small bowel syndrome'. The three cardinal symptoms are persistent diarrhoea, abdominal pain, and weight loss. Localized areas of narrowing and dilatation of the ileum are seen on $\mathrm{X}$-ray, and histology of a thickened portion of ileal wall shows a non-specific granuloma characterized by foreign-body giant cells and lymphocytes, together with clofazimine crystals. Crystals are also deposited in mesenteric lymph nodes. This 'small bowel syndrome' has terminated fatally in a small number of cases in which high dosage has been prolonged in order to control chronic or recurrent ENL reaction, and the lesson is that it would be preferable to reserve clofazimine for the treatment of leprosy in the modern dosage of $300-350 \mathrm{mg} / \mathrm{week}$ and to use much more effective drugs, such as prednisone or thalidomide when control of ENL is necessary. Eosinophilic enteritis has been reported in a female Samoan who was treated with $600 \mathrm{mg}$ daily for 3 years. She presented with abdominal pain and was found to have an eosinophilia $\left(3600 \mathrm{~mm}^{3}\right)$. At laparotomy there were about 20 nodular areas in the wall of upper ileum, and a biopsy showed a dense cellular infiltrate of eosinophils and histiocytes together with clofazimine crystals.

3 RIFAMPICIN (RFP; RMP; RIFAMPIN; RIFADIN; RIMACTANE)

A semi-synthetic derivative of rifamycin B, one of a group of antibiotic compounds produced by Streptomyces mediterranei.

\section{A Side-effects of daily and intermittent administration}

(a) Mild side-effects

The commonest is red colouration of urine. Other side-effects are uncommon and include transient rash, gastro-intestinal symptoms, drowsiness, weakness and dizziness.

(b) Serious side-effects

An uncommon one is hepatitis, and rarer ones are thrombocytopenia, psychosis 
and osteomalacia. Osteomalacia has been reported as a complication of daily therapy and is heralded by a fall in plasma 25-hydroxycole calciferol (25-OHD), and if treatment is continued the patient complains of generalized bone pain and tenderness. X-ray shows generalized demineralization, and bone biopsy confirms the diagnosis. Serious sensitivity reactions, characterized by bullous skin lesions, with or without involvement of oral mucosa, have been reported as rare events and have been diagnosed as Stevens-Johnson syndrome, porphyria cutanea tarda, and pemphigus vulgaris respectively. Long-term antibiotic therapy may deplete the body's supply of vitamin $\mathrm{K}$ through depression of synthesis of this vitamin in the intestinal tract. This can occur when rifampicin is given daily over a long period of time, and if the patient is a pregnant woman she may give birth to an infant with haemorrhagic disease of the newborn. This complication has been reported from Germany where a woman suffering from lepromatous leprosy was treated throughout her pregnancy with multidrug therapy containing rifampicin.

\section{B Side-effects confined to intermittent administration}

These have occurred in the treatment of tuberculosis when rifampicin has been administered once-weekly or twice-weekly, but are very unlikely with oncemonthly treatment of leprosy as recommended by the 1982 WHO Study Group. These include: (1) 'Flu' syndrome. Episodes of fever and malaise, sometimes associated with headache, dizziness and pains in limbs, begin 1-2 hours after each treatment and last up to 8 hours. The syndrome is unlikely during the first 3 months of therapy, but thereafter may occur in up to $20 \%$ of patients given once-weekly dosage. (2) Shock, dyspnoea, haemolytic anaemia and renal failure may rarely complicate intermittent treatment with rifampicin; they may also occur when treatment has been resumed in full dosage after a long interval.

Here I must add a note on the reduced effectiveness of steroid when given concurrently with rifampicin. This is due to rifampicin's ability to stimulate the production of hepatic microsomal enzymes which increase the metabolic degradation of steroid. Similarly, it can impair the effectiveness of oral contraceptives, and this could lead to an undesired pregnancy in a lepromatous woman; worse still, if the woman is given thalidomide to control a severe ENL reaction on the strength that the pill will prevent pregnancy, the consequences could be disastrous. Associated with rifampicin's capacity to counteract the effect of prednisone during ENL reaction is its counteracting effect on endogenous cortisol. This causes no problem when cortisol production is normal, but in patients with adrenal cortical dysfunction it can be dangerous to give rif ampicin. This risk has been highlighted by a recent report of acute adrenal crisis in 2 patients with adrenal insufficiency due to tuberculosis who were treated with rifampicin. 
ETHIONAMIDE (2-ETHYLPYRIDINE-4-CARBOTHIONAMIDE)

(a) Mild side-effects

These include gastro-intestinal symptoms, ptyalism (excessive salivation), a metallic taste, stomatitis, transient rash, acne, headache and dizziness. All are uncommon apart from gastric symptoms.

\section{(b) Serious side-effects}

These are uncommon. (1) Peripheral neuropathy. (2) Hepatitis. Although clinical jaundice is rare, abnormal liver function tests have been described in $15 \%$, and a rise in transaminase is an indication to keep progress under review while continuing treatment. (3) Hypothyroidism. A diffuse enlargement of the thyroid gland is an early sign. (4) Hypoglycaemia. A connection between sleepiness and hypoglycaemia has been observed, indicating that blood sugar levels should be tested in a patient who becomes drowsy. (5) Psychosis. A pellagra-like encephalopathy has been described, with depression, slowed cerebration, personality changes, and difficulty in walking. Tendon reflexes are brisk and plantar responses may be extensor. Response to nicotinamide is dramatic. This syndrome is more likely to occur if ethionamide is given together with isoniazid (INH) because INH interferes with pyridoxine metabolism, and pyridoxine is a co-factor in the synthesis of nicotinamide from tryptophane. (6) Alopecia. A very rare association between alopecia and ethionamide has been reported, but the mechanism is not understood. (7) Endocrine disturbance. Gynaecomastia has been attributed to ethionamide.

5 THiACETAZONE (TBi; AMITHIOZONE; 4-ACETAMINO BENZALDEHyde THIOSEMICARBAZONE)

(a) Mild side-effects

These include gastro-intestinal symptoms, transient rash, dizziness, headache and drowsiness.

(b) Serious side-effects

(1) Skin. Exanthematous skin eruption, exfoliative dermatitis, toxic epidermal necrolysis, and the Stevens-Johnson syndrome have all been encountered, and skin lesions similar to those of lichen planus have been attributed to thiacetazone. (2) Blood. Haemolytic anaemia, agranulocytosis and thrombocytopenia have been reported. (3) Liver. Hepatitis may develop as part of a hypersensitivity syndrome or may occur on its own. It is not uncommon for thiacetazone to cause 
abnormal liver function tests without clinical jaundice, and because of its hepatotoxicity it is inadvisable to give the drug to those with preexisting liver impairment. (4) Ototoxicity. It has been found that streptomycin's capacity to cause disturbance of hearing and balance is enhanced if given with thiacetazone.

A unique quality of thiacetazone is that the frequency and severity of its side-effects are influenced by racial and geographical factors. For example, in some areas of India the drug is well tolerated whereas in others there is a high incidence of serious dermatological complications.

Finally, thiacetazone and ethionamide should not be combined in the treatment of leprosy or tuberculosis because of cross-resistance.

W H JOPLING

[The author has kindly offered to make available a list of references on this subject to anyone who cares to apply to the Editorial Office in Oxford. EDITOR] 